\title{
Mortality of native and invasive ladybirds co-infected by ectoparasitic and entomopathogenic fungi
}

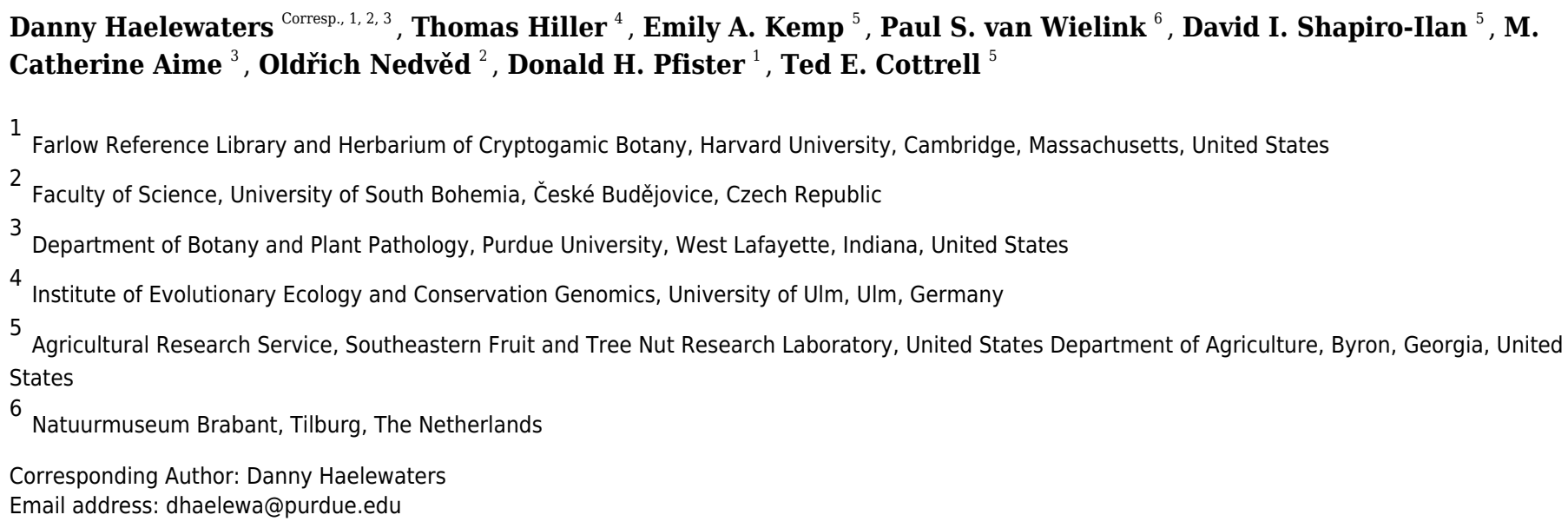

Harmonia axyridis is an invasive alien ladybird in North America and Europe. Studies show that multiple natural enemies are using $\mathrm{Ha}$. axyridis as a new host. However, thus far, no research has been undertaken to study the effects of simultaneous infections of multiple natural enemies on Ha. axyridis. We hypothesized that high thallus densities of the ectoparasitic fungus Hesperomyces virescens on a ladybird weaken the host's defenses, thereby making it more susceptible to infection by other natural enemies. We examined mortality of the North American-native Olla v-nigrum and Ha. axyridis co-infected with $\mathrm{He}$. virescens and an entomopathogenic fungus-either Beauveria bassiana or Metarhizium brunneum. Laboratory assays revealed that He. virescens-infected O. v-nigrum individuals are more susceptible to entomopathogenic fungi, but $\mathrm{Ha}$. axyridis does not suffer the same effects. This is in line with the enemy release hypothesis, which predicts that invasive alien species in new geographic areas experience reduced regulatory effects from natural enemies compared to native species. Considering our results, we can ask how He. virescens affects survival when confronted by other pathogens that previously had little impact on Ha. axyridis. 
1 Mortality of native and invasive ladybirds co-infected

2 by ectoparasitic and entomopathogenic fungi

3

4

5

Danny Haelewaters ${ }^{1,2,3 *}$, Thomas Hiller ${ }^{4}$, Emily A. Kemp ${ }^{5}$, Paul S. van Wielink ${ }^{6}$, David I. Shapiro-Ilan ${ }^{5}$, M. Catherine Aime ${ }^{3}$, Oldřich Nedvěd ${ }^{2}$, Donald H. Pfister ${ }^{1}$, Ted E. Cottrell ${ }^{5}$

${ }^{1}$ Harvard University, Farlow Reference Library and Herbarium of Cryptogamic Botany, Cambridge, Massachusetts, USA

${ }^{2}$ University of South Bohemia, Faculty of Science, České Budějovice, Czech Republic

${ }^{3}$ Purdue University, Department of Botany and Plant Pathology, West Lafayette, Indiana, USA

${ }^{4}$ University of Ulm, Institute of Evolutionary Ecology and Conservation Genomics, Ulm, Germany

${ }^{5}$ United States Department of Agriculture, Agricultural Research Service, Southeastern Fruit and Tree Nut Research Laboratory, Byron, Georgia, USA

${ }^{6}$ Natuurmuseum Brabant, Tilburg/De Kaaistoep, The Netherlands

Corresponding Author:

Danny Haelewaters

Lilly Hall of Life Sciences, 915 W. State Street, West Lafayette, Indiana, 47907, USA

Email address: danny.haelewaters@gmail.com

\section{Abstract}

Harmonia axyridis is an invasive alien ladybird in North America and Europe. Studies show that multiple natural enemies are using $\mathrm{Ha}$. axyridis as a new host. However, thus far, no research has been undertaken to study the effects of simultaneous infections of multiple natural enemies on Ha. axyridis. We hypothesized that high thallus densities of the ectoparasitic fungus

Hesperomyces virescens on a ladybird weaken the host's defenses, thereby making it more susceptible to infection by other natural enemies. We examined mortality of the North Americannative Olla v-nigrum and Ha. axyridis co-infected with He. virescens and an entomopathogenic fungus - either Beauveria bassiana or Metarhizium brunneum. Laboratory assays revealed that $H$. virescens-infected $O$. v-nigrum individuals are more susceptible to entomopathogenic fungi, but Ha. axyridis does not suffer the same effects. This is in line with the enemy release hypothesis, which predicts that invasive alien species in new geographic areas experience reduced regulatory effects from natural enemies compared to native species. Considering our results, we can ask how He. virescens affects survival when confronted by other pathogens that previously had little impact on Ha. axyridis.

\section{Introduction}


40 In nature, hosts may be exploited by more than one natural enemy. These organisms interact with

41

42

43

44

45

46

47

48

49

50

51

52

53

54

55

56

57

58

59

60

61

62

63

64

65

66

67

68

69

70

71

72

73

74

75

76

77

78

79 each other and with their hosts (Furlong \& Pell, 2005). These complex interactions shape the population structure and dynamics of all organisms in the system. Natural enemies also compete with one another, and the impact on the host can be either synergistic, additive, or antagonistic (Shapiro-Ilan et al., 2012). These interactions can be manifested in various aspects of host fitness or mortality. For example, biological control of Drosophila suzukii (Diptera, Drosophilidae), an important pest of fruit and berry crops, can be improved by treatments combining multiple natural enemies, which have an additive effect (Renkema \& Cuthbertson, 2018). At the same time, dual infections (even if causing an increase in host mortality) may be deleterious to one or both pathogens in terms of pathogen growth, fecundity, or other fitness parameters.

Harmonia axyridis (Coleoptera, Coccinellidae), native to eastern Asia, has rapidly increased its global range and is now present on all continents except Antarctica (Roy et al., 2016; CamachoCervantes et al., 2017; Hiller \& Haelewaters, 2019). Even though it has repeatedly been introduced for its beneficial properties as a biological control agent against aphid pests, its negative effects on native ladybird communities in invaded areas (Koch et al., 2006; Honěk et al. 2016; Brown \& Roy, 2018) and on food production (Koch et al., 2006) have raised serious concerns since the early 2000s (Roy et al., 2016). It is now a model organism for studying invasive alien species (Roy \& Wajnberg, 2008; Brown et al., 2018) and it has been listed in Europe as "one of the worst" invasive species (Nentwig et al., 2018). Harmonia axyridis is often reported as a host to several natural enemies. These include parasites (Hesperomyces virescens, Coccipolipus hippodamiae, Parasitylenchus bifurcatus), parasitoids (phorid and tachinid flies, Dinocampus coccinellae, Homalotylus spp., Tetrastichinae spp.), pathogens (bacteria, fungi, nematodes, protozoans), and predators (bugs, lacewings, ladybirds, and spiders) (Garcés \& Williams, 2004; Riddick et al., 2008; Riddick, 2010; Harding et al., 2011; Raak-van den Berg et al., 2014; Haelewaters et al., 2017; Ceryngier et al. 2018). Independent studies show that natural enemies of native ladybirds have recently employed Ha. axyridis as a new host, sometimes simultaneously (Raak-van den Berg et al., 2014; Haelewaters et al., 2017; Ceryngier et al. 2018; Knapp et al., 2019). Review of the effects of parasites, pathogens, and parasitoids of Ha. axyridis shows that they have only limited potential for controlling population densities of their host when acting alone (Roy et al., 2008; Riddick, 2010; Haelewaters et al., 2017; Ceryngier et al., 2018). Thus far, no studies have focused on the effects of infections of multiple natural enemy on Ha. axyridis.

Hesperomyces virescens (Ascomycota, Laboulbeniomycetes, Laboulbeniales) is a common obligate ectoparasite of ladybirds (Roy et al., 2016; Haelewaters et al., 2017). Although known since 1891 , it was shown only recently that He. virescens is in reality a complex of multiple hostspecific species (Haelewaters et al., 2018). Contrary to most multicellular fungi, He. virescens as well as other members of the Laboulbeniales order lack hyphae, instead they form 3-dimensional multicellular thalli by determinate growth (Blackwell et al., 2020). Laboulbeniales, including He.

Peer) reviewing PDF | (2020:04:48281:2:0:CHECK 7 Sep 2020) 
80

81

82

83

84

85

86

87

88

89

90

91

92

93

94

95

96

97

98

99

100

101

102

103

104

105

106

107

108

109

110

111

112

113

114

115

116

117

118

119

virescens, cannot be grown in axenic culture and no asexual stages are known, which makes their study challenging (Haelewaters et al., 2021). Given locally high prevalence of He. virescens on ladybird hosts (Riddick \& Cottrell, 2010; Haelewaters et al., 2017) and the abundance of entomopathogenic fungal strains in the environment (Roy \& Cottrell, 2008), we examined mortality of native and invasive He. virescens-infected ladybirds exposed to either Beauveria bassiana or Metarhizium brunneum (Ascomycota, Sordariomycetes, Hypocreales) (sensu Cottrell \& Shapiro-Ilan, 2003, 2008). Because He. virescens forms a branched, non-septate, rhizoidal haustorium (Weir \& Beakes, 1996) that penetrates the host's exoskeleton and makes contact with the body fluid for nutrient uptake, we hypothesized that high thallus densities with concomitant haustorial formation by He. virescens weaken host defenses, thus increasing the host's susceptibility to infection by other natural enemies. With this experiment, we assess how He. virescens affects ladybird survival when exposed to other natural enemies that alone have little impact on Ha. axyridis and compare results with a North American-native ladybird of similar body size, Olla v-nigrum. If He. virescens - on its own and in combination with other natural enemies - significantly impacts survival of the invasive ladybird but not the native one, then the results of this work could have consequences toward a pest management strategy to control infestations of vineyards and agroecosystems by Ha. axyridis.

\section{Materials \& Methods}

\section{Field collections and laboratory colonies}

Harmonia axyridis and Olla v-nigrum ladybirds were collected for the purpose of establishing laboratory colonies of Hesperomyces-infected and non-infected ladybirds. Specimens were collected at overwintering sites at the 485-ha USDA-ARS, Southeastern Fruit and Tree Nut Research Laboratory, located in Byron, Georgia, USA (32.657792, -83.7383580). Sex and age of field-collected specimens were not determined to reduce dispersal of fungal propagules (Cottrell \& Riddick, 2012). All specimens were brought to the laboratory and housed in individual Petri plates $(10 \mathrm{~cm}$ diam.) with $1 / 3$ of a piece of a cotton dental wick (Deerpack Products, LLC, Miami, Florida) drenched in water for hydration. Ladybirds were housed in environmental chambers at $25 \pm 1{ }^{\circ} \mathrm{C}$ and photoperiod of $14: 10$ (L:D) h. Food was provided $3 \times$ per week in the form of Ephestia kuehniella eggs (Lepidoptera, Pyralidae) and an artificial meat-based diet (Beneficial Insectary, Redding, California). Olla v-nigrum and Ha. axyridis ladybirds were maintained within the Petri plates for $14 \mathrm{~d}$ and $21 \mathrm{~d} 32$, respectively, at which time ladybirds were visually examined for presence of Hesperomyces using a dissecting microscope at 50× magnification. Eggs were harvested from ovipositing ladybirds and used to establish clean (free from fungal growth) laboratory-reared colonies of ladybirds with known age.

\section{Laboratory rearing of ladybirds}

During examination for presence/absence of Hesperomyces, ladybirds were divided into two groups, infected and non-infected. Both groups of ladybirds were placed into plastic rearing containers of $19 \times 13.5 \times 9 \mathrm{~cm}$ (Pioneer Plastics, North Dixon, Kentucky), which were modified 
120

121

122

123

124

125

126

127

128

129

130

131

132

133

134

135

136

137

138

139

140

141

142

143

144

145

146

147

148

149

150

151

152

153

154

155

156

157

158

159

with two $3-\mathrm{cm}$ diameter circular openings, one that was covered by $1 \times 1 \mathrm{~mm}$ mesh to allow for air flow; and the second that was covered with a removable \#7 rubber stopper to allow for feeding routinely as well as adding newly emerged laboratory-reared ladybirds. Routine maintenance included transferring ladybirds into fresh rearing containers at the end of each $7 \mathrm{~d}$ period, which included nutrient supplementations of laboratory-reared yellow pecan aphids, Monelliopsis pecanis (Hemiptera, Aphididae).

The first laboratory generation of adults emerged about one month after placement in rearing containers. Emerging adults were placed into fresh rearing containers and stored into a separate incubator $\left(25 \pm 1{ }^{\circ} \mathrm{C}, 14: 10(\mathrm{~L}: \mathrm{D}) \mathrm{h}\right)$ for 7 days. Similar to field-captured $O$. v-nigrum and $\mathrm{Ha}$. axyridis, M. pecanis aphids were used as a diet augmentation. As the study progressed, we also incorporated black pecan aphids, Melanocallis caryaefoliae (Hemiptera, Aphididae), in the ladybird diet $(3 \times$ per week).

\section{Artificial transmissions of Hesperomyces}

Not only did we need the ladybirds for our experiments to be of the same age, we also needed to artificially infect a subset of these "clean" laboratory-grown, adult ladybirds with Hesperomyces virescens. Exposure to Hesperomyces was conducted via tumbling of the field-captured 'source' ladybirds (infected with Hesperomyces) with randomly selected laboratory-reared 'target' ladybirds (Cottrell \& Shapiro-Ilan, 2008). A total of 25 target ladybirds were mixed with 5 Hesperomyces-infected source ladybirds in a $1.6 \times 5.8 \mathrm{~cm}$ glass tube, which was placed on a hotdog roller (Nostalgia Electrics, Green Bay, Wisconsin) for $5 \mathrm{~min}$. This procedure was repeated for at least 160 target ladybirds of both species. We only performed intra-specific artificial transmissions of Hesperomyces, meaning from source Ha. axyridis to target Ha. axyridis and from source $O$. v-nigrum to target $O$. v-nigrum. Both Hesperomyces-exposed target ladybirds and clean (unexposed) ladybirds were fed a diet of $M$. pecanis aphids for $24 \mathrm{~h}$. We did a second tumbling experiment using randomly selected emerged adults from the second cohort of laboratory-reared colonies. More tumbling experiments were performed to increase quantities of Hesperomyces-infected ladybirds, but source/target numbers were changed to 100/40.

To reduce competition for food, ladybirds from all laboratory colonies were transferred from the plastic rearing containers to 14-cm diameter Petri plates. Ladybirds were provided with water ad libitum, E. kuehniella eggs, and artificial meat-based diet. Finally, for assay preparation, the ladybirds were transferred back to clean $19 \times 13.5 \times 9 \mathrm{~cm}$ plastic rearing containers by species.

\section{Dual fungal infections assay}

Within 24 hours preceding the assay, 160 non-infected and 160 Hesperomyces-infected ladybirds of each species (Ha. axyridis and O. v-nigrum) were each placed into sterile test tubes, one individual per test tube. Test tubes were then closed with a sterile foam stopper to prevent ladybirds from escaping while allowing for air flow. Infected ladybirds were divided into 
160

161

162

163

164

165

166

167

168

169

170

171

172

173

174

175

176

177

178

179

180

181

182

183

184

185

186

187

188

189

190

191

192

193

194

195

196

197

198

199

categories according to numbers of thalli per specimen. Because the assay would assess potential interactions between fungal infections, we aimed at selecting heavily Hesperomyces-infected ladybirds; as a baseline, we only used specimens in our bioassays with 14 or more thalli each.

The assay started by pipetting a $1 \mathrm{~mL}$ of $2.5 \times 10^{5}$ conidia/mL suspension to each test tube (Cottrell \& Shapiro-Ilan, 2003, 2008). Treatments included native B. bassiana (native Bb), a commercial B. bassiana strain (GHA Bb; Mycotrol ES, Mycotech, Butte, Montana), M. brunneum strain F52 (Mb, isolated from a tortricid moth, Austria 1971; Novozymes, Franklinton, North Carolina), and double-distilled water $\left(\mathrm{ddH}_{2} \mathrm{O}\right)$ as a control treatment. Ladybirds were submerged and swirled for $5 \mathrm{~s}$, after which the suspension was removed again using a pipette and each ladybird was placed into a $6 \mathrm{~cm}$-diameter Petri plate. Any remaining droplets of excess suspension was removed by touching only the droplet with a Kimwipe tissue (Kimtech Science Brand, Kimberly-Clark Worldwide, Roswell, Georgia). Petri plates with treated ladybirds were placed into an incubator $\left(25 \pm 1{ }^{\circ} \mathrm{C}, 14: 10\right.$ (L:D) h). Food and cotton rolls drenched in water were provided ad libitum, and Petri plates were replaced as needed in all treatments and replications simultaneously. Ladybirds were observed for mortality and entomopathogeninduced mycosis at day 14 . During assay \#1, we made daily observations for ladybird mortality and mycosis. Upon death of a given ladybird, ample water was added to the cotton roll to provide moisture for entomopathogen growth and Parafilm was applied around the Petri plate to prevent spreading of the fungus. Deaths of ladybirds and visual confirmations of mycosis were recorded.

We performed 8 different treatments for each ladybird species: 1) He. virescens-positive + native $\mathrm{Bb}, 2)$ He. virescens-positive $+\mathrm{GHA} \mathrm{Bb}, 3)$ He. virescens-positive $+\mathrm{Mb}$, 4) He. virescenspositive $+\mathrm{ddH}_{2} \mathrm{O}$ (control), 5) He. virescens-negative + native $\left.\mathrm{Bb}, 6\right)$ He. virescens-negative + $\mathrm{GHA} \mathrm{Bb}, 7) \mathrm{He}$. virescens-negative $+\mathrm{Mb}$, and 8) He. virescens-negative $+\mathrm{ddH}_{2} \mathrm{O}$ (double control). In a single assay, we replicated every treatment 3 or 4 times. We performed the entire assay with all treatments and replicated 3 times, using 6-10 ladybirds for each treatment. Note that $M$. brunneum treatments were used only in assay \#3 (Table S1). Over all assays done during this study, we used 1,289 specimens of ladybirds (667 O. v-nigrum and 622 Ha. axyridis) (Table S2).

\section{Statistical analyses}

All statistical analyses were performed in the R language and open-access environment for statistical computing v.3.5.0. We used generalized linear mixed models (function glmer(), Rpackage lme4; Bates et al., 2015) to analyze the effect of the different treatments (GHA Bb, native $\mathrm{Bb}, \mathrm{Mb}$ ) on the survival of Ha. axyridis and $O . v$-nigrum in relation to prior infection with Hesperomyces. We modeled the binary response variable survival (alive/dead) of each ladybird individual for both host species separately, and used Hesperomyces infection status as well as the interaction of Hesperomyces infection status with treatment as explaining variables. Further, to 
200

201

202

203

204

205

206

207

208

209

210

211

212

213

214

215

216

217

218

219

220

221

222

223

224

225

226

227

228

229

230

231

232

233

234

235

236

237

238

239

correct for variation within replicates and assays, we included the random effect of treatment nested in replicate nested in assay. We compared our candidate models to a respective Nullmodel using likelihood ratio tests and, furthermore, calculated pseudo $\mathrm{R}^{2}$-values (function $\mathrm{r} 2($ ), $\mathrm{R}$ package sjstats; Lüdecke, 2018) to evaluate model fit. To visualize the modeling results and obtained model estimates as forest plots, we used the function plot_model() implemented in the R package sjstats (Lüdecke, 2018). For assay \#1, we further fitted Kaplan-Meier curves to daily mortality data and tested for significant differences in mortality between ladybird species using the function survfit() of the R package survival (Therneau \& Lumley, 2019).

\section{Results}

Our candidate models for both host species $\mathrm{Ha}$. axyridis and $\mathrm{O}$. v-nigrum were significantly better at explaining survival relative to chance variation (Chi-squared test, $\chi^{2}=156.7, P<0.001$; $\chi^{2}=153.0, P<0.001$, respectively). The overall model fit was high for both candidate models (Ha. axyridis: Nagelkerke's $\mathrm{R}^{2}=0.40 ;$ O. v-nigrum: Nagelkerke's $\mathrm{R}^{2}=0.53$ ) suggesting the variance is well described by our applied models.

We found a significant negative effect on ladybird survival of the M. brunneum treatment on He. virescens-negative Ha. axyridis (Fig. 1, Table 1), whereas B. bassiana treatments did not affect the survival of $H e$. virescens-negative individuals. Infection with He. virescens significantly affected Ha. axyridis survival over all treatments (Fig. 1). However, there was no additional effect detectable among co-infection treatments for He. virescens-positive ladybirds (Table 1). Each treatment applied to $O$. v-nigrum had a significantly negative effect on the survival for both He. virescens-negative and -positive ladybirds (Fig. 1, Table 1). Finally, we found an additional negative effect of all co-infection treatments on the survival of He. virescens-positive $O$. $v$ nigrum (Fig. 1, Table 1). These results suggest that there is no effect of dual infections on Ha. axyridis, whereas $O$. v-nigrum is highly affected by simultaneous exposure to both He. virescens and an entomopathogenic fungus. Percentages of ladybird mortality by treatment are also presented in tabulated form in Table S3.

When comparing the daily survival of Ha. axyridis and $O$. v-nigrum, no significant differences were found in Hesperomyces-positive only treatments (log rank test, $P=0.4$ ). However, when co-infected $O$. v-nigrum showed a significantly lower survival compared to Ha. axyridis for GHA and native $B$. bassiana strains (log rank test, $P=0.0014$ and $P<0.001$, respectively). Fig. 2 shows how survival is significantly different between the two ladybird species when coinfected with both Hesperomyces and Beauveria bassiana (GHA and native).

\section{Discussion}

Research on the additive effects of multiple natural enemies on a given host is rare, likely because of the complexity involved in designing robust bioassays that include all partners of the system. Combining the natural enemies Orius insidiosus (Hemiptera, Anthocoridae) and 
240 Heterorhabditis bacteriophora (Rhabditida, Heterorhabditidae) resulted in the largest decline in 241 larvae of Drosophila suzukii (Renkema \& Cuthbertson, 2018), which causes major economic 242 losses to fruit crops in its invasive range, spanning North and South America and Europe (Lee et 243 al., 2011). The addition of $O$. insidiosus resulted in 50\% fewer $D$. suzukii larvae compared to 244 treatment with only H. bacteriophora. Plutella xylostella (Lepidoptera, Plutellidae), an important 245 cosmopolitan pest of brassicaceous crops, offers another example. This organism shows 246 resistance to almost all chemical insecticides (Sarfraz et al., 2005). Pandora blunckii and

247 Zoophthora radicans (Zoopagomycota, Entomophthoromycetes, Entomophthorales) both infect $248 P$. xylostella in the field. In co-inoculation studies with Pa. blunckii and Z. radicans in $P$. 249 xylostella larvae, larval cadavers (three days post mortality) were most frequently found with 250 conidia of a single entomopathogen, usually the one that had been inoculated first (prior 251 "residency") — meaning that the other species was excluded (Sandoval-Aguilar et al., 2015). In 252 general, the presence of competing species in the same host resulted in a decreased proportion of 253 254 $P$. xylostella larvae that were infected compared to single inoculations.

258

259

260

261

262

263

264

265

266

267

268

269

270

271

272

273

274

275

276

277

278

279

Regarding Ha. axyridis, the following co-infections of natural enemies have been observed in nature: He. virescens + Coccipolipus hippodamiae mites (Acarina, Podapolipidae) in the USA, Austria, and the Netherlands (Christian, 2001; Riddick, 2010; Raak-van den Berg et al., 2014) and He. virescens + Parasitylenchus bifurcatus nematodes (Nematoda, Allantonematidae) in the Czech Republic, Germany, and the Netherlands (Raak-van den Berg et al., 2014; Haelewaters et al., 2017; Herz \& Kleespiel, 2012). Given the status of Ha. axyridis as an invasive alien species, these findings demand a better understanding of interactions among the different natural enemies and their potential role in limiting populations of Ha. axyridis. To date, bioassays to determine mortality of ladybirds induced by infection by one or more natural enemies have not yet been performed. Likewise, bioassays including Laboulbeniales have only been carried out in one study (Konrad et al., 2015).

When we started this study, He. virescens was considered a single species with multiple ladybird hosts, potentially with multiple strains that infect only a single species, or closely related ones (Cottrell \& Riddick, 2012). It was recently shown that He. virescens is a complex of multiple species, each with its own ladybird host (Haelewaters et al., 2018), which calls for caution in reviewing reports from the extensive body of literature on Hesperomyces findings (summarized in Haelewaters \& De Kesel, 2017). This also means that isolates of He. virescens from $\mathrm{Ha}$. axyridis and $O$. $v$-nigrum in fact represent two different species of Hesperomyces. In other words, the experiments in the current study allow us to make comparisons between two host species, each with their own specific fungal parasite. Future experiments are needed to further disentangle these interactions. Even though horizontal transmission of Hesperomyces among ladybird species is rare (Cottrell \& Riddick, 2012), we should try to infect Ha. axyridis and O. vnigrum ladybirds with the species of Hesperomyces specific to Olla and Harmonia, respectively, perform bioassays, and compare mortality rates under different treatments with our current

Peer) reviewing PDF | (2020:04:48281:2:0:CHECK 7 Sep 2020) 
280

281

282

283

284

285

286

287

288

289

290

291

292

293

294

295

296

297

298

299

300

301

302

303

304

305

306

307

308

309

310

311

312

313

314

315

316

317

318

319

results. Analyzing interactions among natural enemies only make sense when the taxa considered represent single biological species.

We found a significant negative effect of Hesperomyces-only infection on the survival of both ladybird hosts (Fig. 1a, Table S3). Previous work has shown Hesperomyces infections to result in decreased mating frequency of female ladybirds, lower (male) survival rates in winter, and impeded sensing ability and flexibility of legs in heavily infected ladybirds (Nalepa \& Weir, 2007; Riddick, 2010; Haelewaters et al., 2017). One study implicated parasitism by He. virescens as the cause of late summer mortality of Chilocorus bipustulatus ladybirds (Kamburov et al., 1967) but this was later disputed based on controlled laboratory experiments (Applebaum et al., 1971). Our research is the first to explicitly link Hesperomyces infection with increased ladybird mortality.

Our findings on the effects of Hesperomyces on ladybird survival provided a unique opportunity for setting up dual infection assays - the first such experiments to be conducted on ladybirds. When first infected with He. virescens and then exposed to either B. bassiana or M. brunneum, Ha. axyridis mortality was not increased. This result was unexpected. We had hypothesized that $H a$. axyridis with high thallus densities of He. virescens would have lowered host defenses against other pathogens. In contrast, the mechanism fostering low susceptibility of Ha. axyridis to entomopathogenic fungi (Cottrell \& Shapiro-Ilan, 2003; Knapp et al., 2019) is not compromised by infection with He. virescens. Similarly, infection of O. v-nigrum by He. virescens-only increased mortality but - in contrast to Ha. axyridis - there was significantly higher mortality when co-infected by entomopathogenic fungi. Differential susceptibility to entomopathogenic fungi was reported by Cottrell \& Shapiro-Ilan (2003), who showed that native $B$. bassiana was pathogenic to $O$. v-nigrum but not to Ha. axyridis. We confirm these results regarding the native strain but we also found the same differential pattern for the GHA strain of B. bassiana, whereas in the earlier study this strain was reported to be pathogenic to neither ladybird species (Cottrell \& Shapiro-Ilan, 2003). It is perhaps surprising that we detect the GHA strain to be pathogenic to native ladybirds in contrast to the previous results, but ladybird populations may become more susceptible over time for various reasons and natural enemies also become better adapted (Knapp et al., 2019). We note that differential susceptibility has also been reported for entomopathogenic nematodes - again, Ha. axyridis was less susceptible compared to O. v-nigrum (Shapiro-Ilan \& Cottrell, 2005).

In addition, our data are the first account of differential susceptibility to M. brunneum between the invasive Ha. axyridis and the native O. v-nigrum. Whereas infection with M. brunneum had a significantly negative effect on the survival of He. virescens-negative Ha. axyridis, this effect was not visible in the dual infection treatment. The infection with Laboulbeniales probably decreased the susceptibility of Ha. axyridis to infection by M. brunneum, similar to the findings of Konrad et al. (2015). These authors found that Laboulbenia-infected Lasius neglectus ants 
320 (Hymenoptera, Formicidae) showed a decreased susceptibility to Metarhizium brunneum. This

321

322

323

324

325

326

327

328

329

330

331

332

333

334

335

336

337

338

339

340

341

342

343

344

345

346

347

348

349

350

351

352

353

354

355

356

357

358

359

protection against Metarhizium was positively correlated with parasite load. Information on the parasite load of He. virescens on ladybirds in nature is nonexistent. In our bioassays, we selected ladybirds bearing 14 or more fungal thalli as He. virescens-positive specimens. Previous work from a long-term ATBI project in the Netherlands (van Wielink, 2017) points at an average of $19.8 \pm 4.9$ thalli and a maximum of 129 thalli per Ha. axyridis specimen $(n=270)$. No such data are available for $O$. v-nigrum. In other words, based on the available information, the artificial parasite load in our bioassays seems to closely mimic the natural conditions.

Our results provide direct support for the enemy release hypothesis (Jeffries \& Lawton, 1984). This hypothesis is illustrative for the success of invasive alien species and stipulates that an invasive species in new geographic regions will experience reduced regulatory effects from natural enemies compared to native species, resulting in increased population growth of the invasive species Colautti et al., 2004; Roy et al., 2011). However, invasions are dynamic (Schultheis et al., 2015; Haelewaters et al., 2017) and this escape-from-enemies could be lost as invasive species acquire new enemies over time (Hokkanen \& Pimentel, 1989). Support for enemy release explaining the success of Ha. axyridis has come from two studies that reported decreased susceptibility of Ha. axyridis to entomopathogenic fungi (Cottrell \& Shapiro-Ilan, 2003) and entomopathogenic nematodes (Shapiro-Ilan \& Cottrell, 2005) compared to the native American ladybird species. Our work adds another level of complexity by the addition of a second natural enemy to the interactions. Again, we find differential susceptibility between the invasive and native ladybird species - with a reduced regulatory effect of the tested natural enemies on Ha. axyridis.

\section{Conclusions}

In this paper, we show a negative effect of infection by Hesperomyces virescens on the survival of both Harmonia axyridis and Olla v-nigrum ladybirds (Fig. 1a). This is the first study to unequivocally link Hesperomyces infection with increased host mortality and only the second to perform bioassays with hosts co-infected with Laboulbeniales and a second entomopathogenic fungus (Konrad et al., 2015). However, the susceptibility to a secondary entomopathogenic fungus was only elevated in the native American ladybird species (O. v-nigrum), whereas the globally invasive $H a$. axyridis showed no significant increase in mortality when co-infected with either Beauveria bassiana or Metarhizium brunneum (Figs. 1, 2). These findings are consistent with the enemy release hypothesis (Jeffries \& Lawton, 1984) and highlight the difficulty in controlling this invasive alien species. Future studies are needed to elaborate population-specific effects on native and commercial strains of entomopathogenic fungi used in pest control.

\section{Acknowledgements}

The authors would like to thank Merry Bacon and Chace Morrill (USDA, ARS, Byron, Georgia) for technical assistance.

Peer) reviewing PDF | (2020:04:48281:2:0:CHECK 7 Sep 2020) 


\section{References}

362 S. W. Applebaum, R. Kfir, U. Gerson, U. Tadmor. Studies on the summer decline of Chilocorus

363

364

365

366

367

368

369

370

371

372

373

374

375

376

377

378

379

380

381

382

383

384

385

386

387

388

389

390

391

392

393

394

395

396

397

398

bipustulatus in citrus groves of Israel. Entomophaga 16, 433-444 (1971). doi:10.1007/BF02370924

D. Bates, M. Maechler, B. Bolker, S. Walker. Fitting linear mixed-effects models using lme4. J. Stat. Softw. 67, 1-48 (2015). doi:10.18637/jss.v067.i01

M. Blackwell, D. Haelewaters, D. H. Pfister. Laboulbeniomycetes: Evolution, natural history, and Thaxter's final word. Mycologia, in press (2020). doi:10.1080/00275514.2020.1718442 P. M. J. Brown, H. E. Roy. Native ladybird decline caused by the invasive harlequin ladybird Harmonia axyridis: evidence from a long-term field study. Insect Conserv. Divers. 11, 230-239 (2018). doi:10.1111/icad.12266

P. M. J. Brown, D. B. Roy, C. Harrower, H. J. Dean, S. L. Rorke, H. E. Roy. Spread of a model invasive alien species, the harlequin ladybird Harmonia axyridis in Britain and Ireland. Sci. Data 5, 180239 (2018). doi:10.1038/sdata.2018.239

M. Camacho-Cervantes, A. Ortega-Iturriaga, E. del-Val. From effective biocontrol agent to successful invader: the harlequin ladybird (Harmonia axyridis) as an example of good ideas that could go wrong. PeerJ 5, e3296 (2017). doi:10.7717/peerj.3296

P. Ceryngier, O. Nedvěd, A. A. Grez, E. W. Riddick, H. E. Roy, G. San Martin, T. Steenberg, P. Veselý, T. Zaviezo, Á Zúñiga-Reinoso, D. Haelewaters. Predators and parasitoids of the

harlequin ladybird, Harmonia axyridis, in its native range and invaded areas. Biol. Invasions 20, 1009-1031 (2018). doi:10.1007/s10530-017-1608-9

E. Christian. The coccinellid parasite Hesperomyces virescens and further species of the order

Laboulbeniales (Ascomycotina) new to Austria. Ann. Naturh. Mus. Wien 103 B, 599-603 (2001).

R. I. Colautti, A. Ricciardi, I. A. Grigorovich, H. J. MacIsaac. Is invasion success explained by the enemy release hypothesis? Ecol. Lett. 7, 721-733 (2004). doi:10.1111/j.1461-

0248.2004.00616.x

T. E. Cottrell, E. W. Riddick EW. Limited transmission of the ectoparasitic fungus

Hesperomyces virescens between lady beetles. Psyche (2012). doi:10.1155/2012/814378

T. E. Cottrell, D. I. Shapiro-Ilan. Susceptibility of a native and an exotic lady beetle (Coleoptera: Coccinellidae) to Beauveria bassiana. J. Invert. Pathol. 84, 137-144 (2003). doi:10.1016/j.jip.2003.09.003

T. E. Cottrell, D. I. Shapiro-Ilan. Susceptibility of endemic and exotic North American ladybirds (Coleoptera: Coccinellidae) to endemic fungal entomopathogens. Eur. J. Entomol. 105, 455-460 (2008). doi:10.14411/eje.2008.058

M. J. Furlong, J. K. Pell. Interactions between entomopathogenic fungi and other arthropods natural enemies. In: Insect-fungal associations, ecology and evolution, F. E. Vega, M. Blackwell, Eds. (Oxford University Press, 2005), pp. 51-73.

Peer) reviewing PDF | (2020:04:48281:2:0:CHECK 7 Sep 2020) 
399 S. Garcés, R. Williams. First record of Hesperomyces virescens Thaxter (Laboulbeniales:

400 Ascomycetes) on Harmonia axyridis (Pallas) (Coleoptera: Coccinellidae). J. Kansas Entomol.

401 Soc. 77, 156-158 (2004). doi:10.2317/0304.18.1

402 D. Haelewaters, M. Blackwell, D. H. Pfister. Laboulbeniomycetes: Intimate fungal associates of

403 arthropods. An. Rev. Entomol. 66 (2021). doi:10.1146/annurev-ento-013020-013553

404 D. Haelewaters, A. De Kesel. De schimmel Hesperomyces virescens, een natuurlijke vijand van 405 lieveheersbeestjes. Entomol. Ber. 77, 106-118 (2017).

406 D. Haelewaters, A. De Kesel, D. H. Pfister. Integrative taxonomy reveals hidden species within a 407 common fungal parasite of ladybirds. Sci. Rep. 8, 15966 (2018). doi:10.1038/s41598-018-34319$408 \quad 5$

409 D. Haelewaters, S. Y. Zhao, S. Clusella-Trullas, T. E. Cottrell, A. De Kesel, L. Fiedler, A. Herz, 410 H. Hesketh, C. Hui, R. G. Kleespies, J. E. Losey, I. A. Minnaar, K. M. Murray, O. Nedvěd, W. 411 P. Pfliegler, C. L. Raak-van den Berg, E. W. Riddick, D. I. Shapiro-Ilan, R. R. Smyth, T. 412 Steenberg, P. S. van Wielink, S. Viglášova, Z. Zhao, P. Ceryngier, H. E. Roy. Parasites of 413 Harmonia axyridis: current research and perspectives. BioControl 62, 355-371 (2017). 414 doi:10.1007/s10526-016-9766-8

415 S. Harding, G. O. Poinar, D. V. Dimitrova, T. Steenberg. Parasitylenchus sp. (Tylenchomorpha: 416 Allantonematidae) parasitizing field populations of Harmonia axyridis (Coleoptera:

417 Coccinellidae). Eur. J. Entomol. 108, 487-488 (2011). doi:10.14411/eje.2011.062

418 A. Herz, R. G. Kleespies. Occurrence of natural enemies in different populations of the invasive 419 ladybird Harmonia axyridis (Pallas, 1771) (Coleoptera, Coccinellidae) in Germany. Mitt.

420 Deutsch Ges. allg. angew. Entomol. 18, 201-206 (2012).

421 T. Hiller, D. Haelewaters. A case of silent invasion: citizen science confirms the presence of

422 Harmonia axyridis (Coleoptera, Coccinellidae) in Central America. Plos One 14, e0220082 423 (2019). doi:10.1371/journal.pone.0220082

424 H. M. T. Hokkanen, D. Pimentel. New associations in biological control: theory and practice.

425 Can. Entomol. 121, 829-840 (1989). doi:10.4039/Ent121829-10

426 A. Honěk, Z. Martinkova, A. F. G. Dixon, H. E. Roy, S. Pekar. Long-term changes in

427 communities of native coccinellids: population fluctuations and the effect of competition from an 428 invasive non-native species. Insect Conserv. Divers. 9, 202-209 (2016). doi:10.1111/icad.12158

429 M. J. Jeffries, J. H. Lawton. Enemy free space and the structure of ecological communities. Biol. 430 J. Linn. Soc. 23, 269-286 (1984). doi:10.1111/j.1095-8312.1984.tb00145.x

431 S. S. Kamburov, D. J. Nadel, R. Kenneth. Observations on Hesperomyces virescens Thaxter 432 (Laboulbeniales), a fungus associated with premature mortality of Chilocorus bipustulatus $\mathrm{L}$. in 433 Israel. Israel J. Agric. Res. 17, 131-134 (1967).

434 M. Knapp, M. Řeřicha, S. Maršíková, F. Harabiš, T. Kadlec, O. Nedvěd, T. Teder. Invasive host 435 caught up with a native parasitoid: field data reveal high parasitism of Harmonia axyridis by 436 Dinocampus coccinellae in Central Europe. Biol. Invasions 21, 2795-2802 (2019).

437 doi:10.1007/s10530-019-02027-4 
438 R. L. Koch, R. C. Venette, W. D. Hutchison. Invasions by Harmonia axyridis (Pallas)

439 (Coleoptera: Coccinellidae) in the Western Hemisphere: implications for South America.

440 Neotrop. Entomol. 35, 421-434 (2006). doi:10.1590/S1519-566X2006000400001

441 M. Konrad, A. V. Grasse, S. Tragust, S. Cremer. Antipathogen protection versus survival costs

442 mediated by an ectosymbiont in an ant host. Proc. Royal Soc. B 282, 20141976 (2015).

443 doi:10.1098/rspb.2014.1976

444 J. C. Lee, D. J. Bruck, A. J. Dreves, C. Ioriatti, H. Vogt, P. Baufeld, In focus: Spotted wing 445 drosophila, Drosophila suzukii, across perspectives. Pest Manag. Sci. 67, 1349-1351 (2011).

446 doi:10.1002/ps.2271

447 D. Lüdecke. sjstats: Statistical functions for regression models. R package version 0.15.0.

448 https://CRAN.R-project.org/package=sjstats (2018).

449 C. A. Nalepa, A. Weir. Infection of Harmonia axyridis (Coleoptera: Coccinellidae) by

450 Hesperomyces virescens (Ascomycetes: Laboulbeniales): role of mating status and aggregation

451 behavior. J. Invertebr. Pathol. 94, 196-203 (2007). doi:10.1016/j.jip.2006.11.002

452 W. Nentwig, S. Bacher, S. Kumschick, P. Pyšek, M. Vilà. More than "100 worst" alien species

453 in Europe. Biol. Invasions 20, 1611-1621 (2018). doi:10.1007/s10530-017-1651-6

454 C. L. Raak-van den Berg, P. S. van Wielink, P. W. de Jong, G. Gort, D. Haelewaters, J. Helder,

455 G. Karssen, J. C. van Lenteren. Invasive alien species under attack: natural enemies of Harmonia

456 axyridis in the Netherlands. BioControl 59, 229-240 (2014). doi:10.1007/s10526-014-9561-3

457 J. M. Renkema, A. G. S. Cuthbertson. Impact of multiple natural enemies on immature

458 Drosophila suzukii in strawberries and blueberries. BioControl 63, 719-728 (2018).

459 doi:10.1007/s10526-018-9874-8

460 E. W. Riddick. Ectoparasitic mite and fungus on an invasive lady beetle: Parasite coexistence

461 and influence on host survival. Bull. Insectol. 63, 13-20 (2010).

462 E. W. Riddick, T. E. Cottrell. Is the prevalence and intensity of the ectoparasitic fungus

463 Hesperomyces virescens related to the abundance of entomophagous coccinellids? Bull. Insectol.

464 63, 71-78 (2010).

465 E. W. Riddick, T. E. Cottrell, K. A. Kidd. Natural enemies of the Coccinellidae: Parasites,

466 pathogens, and parasitoids. Biol. Control 51, 306-312 (2008).

467 doi:10.1016/j.biocontrol.2009.05.008

468 H. E. Roy, P. M. J. Brown, T. Adriaens, N. Berkvens, I. Borges, S. Clusella-Trullas, R. F.

469 Comont, P. De Clercq, R. Eschen, A. Estoup, E. W. Evans, B. Facon, M. M. Gardiner, A. Gil, A.

470 A. Grez, T. Guillemaud, D. Haelewaters, A. Herz, A. Honek, A. G. Howe, C. Hui, W. D.

471 Hutchison, M. Kenis, R. L. Koch, J. Kulfan, L. Lawson Handley, E. Lombaert, A. Loomans, J.

472 Losey, A. O. Lukashuk, D. Maes, A. Magro, K. M. Murray, G. San Martin, Z. Martinkova, I.

473 Minnaar, O. Nedved, M. J. Orlova-Bienkowskaja, W. Rabitsch, H. P. Ravn, G. Rondoni, S. L.

474 Rorke, S. K. Ryndevich, M.-G. Saethre, J. J. Sloggett, A. O. Soares, R. Stals, M. C. Tinsley, A.

475 Vandereycken, P. van Wielink, S. Viglášová, P. Zach, T. Zaviezo, Z. Zhao. The harlequin

476 ladybird, Harmonia axyridis: global perspectives on invasion history and ecology. Biol.

477 Invasions 18, 997-1044 (2016). doi:10.1007/s10530-016-1077-6 
478 H. E. Roy, P. M. J. Brown, P. Rothery, R. L. Ware, M. E. N. Majerus. Interactions between the 479 fungal pathogen Beauveria bassiana and three species of coccinellid: Harmonia axyridis, 480 Coccinella septempunctata and Adalia bipunctata. BioControl 53, 265-276 (2008).

481 doi:10.1007/s10526-007-9122-0

482 H. E. Roy, T. E. Cottrell. Forgotten natural enemies: Interactions between coccinellids and 483 insect-parasitic fungi. Eur. J. Entomol. 105, 391-398 (2008). doi:10.14411/eje.2008.049

484 H. E. Roy, L-J. Lawson Handley, K. Schönrogge, R. L. Poland, B. V. Purse. Can the enemy 485 release hypothesis explain the success of invasive alien predators and parasitoids? BioControl 486 56, 451-468 (2011). doi:10.1007/s10526-011-9349-7

487 H. E. Roy, E. Wajnberg. From biological control to invasion: the ladybird Harmonia axyridis as 488 a model species. BioControl 53, 1-4 (2008). doi:10.1007/s10526-007-9127-8

489 J. A. Sandoval-Aguilar, A. W. Guzmán-Franco, J. K. Pell, S. J. Clark, R. Alatorre-Rosas, M. T. 490 Santillán-Galicia, G. Valdovinos-Ponce. Dynamics of competition and co-infection between 491 Zoophthora radicans and Pandora blunckii in Plutella xylostella larvae. Fungal Ecol. 17, 1-9 492 (2015). doi:10.1016/j.funeco.2015.04.003

493 M. Sarfraz, A. B. Keddie, L. M. Dosdall. Biological control of the diamondback moth, Plutella 494 xylostella: A review. Biol. Sci. Technol. 15, 763-789 (2005). doi:10.1080/09583150500136956 495 E. H. Schultheis, A. E. Berardi, J. A. Lau. No release for the wicked: enemy release is dynamic 496 and not associated with invasiveness. Ecology 96, 2446-2457 (2015). doi:10.1890/14-2158.1

497 D. I. Shapiro-Ilan, D. J. Bruck, L. A. Lacey. Principles of epizootiology and microbial control.

498 In: Insect pathology, 2nd edition, F. E. Vega, H. K. Kaya, Eds. (Elsevier, 2012), pp 29-72.

499 doi:10.1016/B978-0-12-384984-7.00003-8

500 D. I. Shapiro-Ilan, T. E. Cottrell. Susceptibility of lady beetles (Coleoptera: Coccinellidae) to 501 entomopathogenic nematodes. J. Invert. Pathol. 89, 150-156 (2005).

502 doi:10.1016/j.jip.2005.04.002

503 T. Therneau, T. Lumley. Package 'survival', version 2.44-1.1 https://cran.r-

504 project.org/web/packages/survival/survival.pdf (2019).

505 P. S. van Wielink. Harmonia axyridis (Coleoptera: Coccinellidae): 13 jaar gevolgd met

506 lichtvangsten in De Kaaistoep, Noord-Brabant. Entomol. Ber. 77: 97-105 (2017).

507 A. Weir, G. W. Beakes. Correlative light- and scanning electron microscope studies on the

508 developmental morphology of Hesperomyces virescens. Mycologia 88, 677-693 (1996).

509 doi:10.1080/00275514.1996.12026705 


\section{Figure 1}

Results of treatment effects on native and invasive ladybirds.

A) Percentages of ladybird mortality by treatment (left Harmonia axyridis, right Olla v-

nigrum). B), C) Forest plots illustrating the results of our modelling approach showing the treatment effects on survival of ladybirds (negative effect in red (odds ratio $<1$ ), positive effect in blue (odds ratio $>1$ ); $* * P<0.01,{ }^{* * *} P<0.001$ ). B) Olla v-nigrum. C) Harmonia axyridis. Photo credits: Olla v-nigrum, Roberto Güller (Flickr); Harmonia axyridis, Andreas Trepte (www.photo-natur.net). Drawings of Hesperomyces thalli by André De Kesel. 


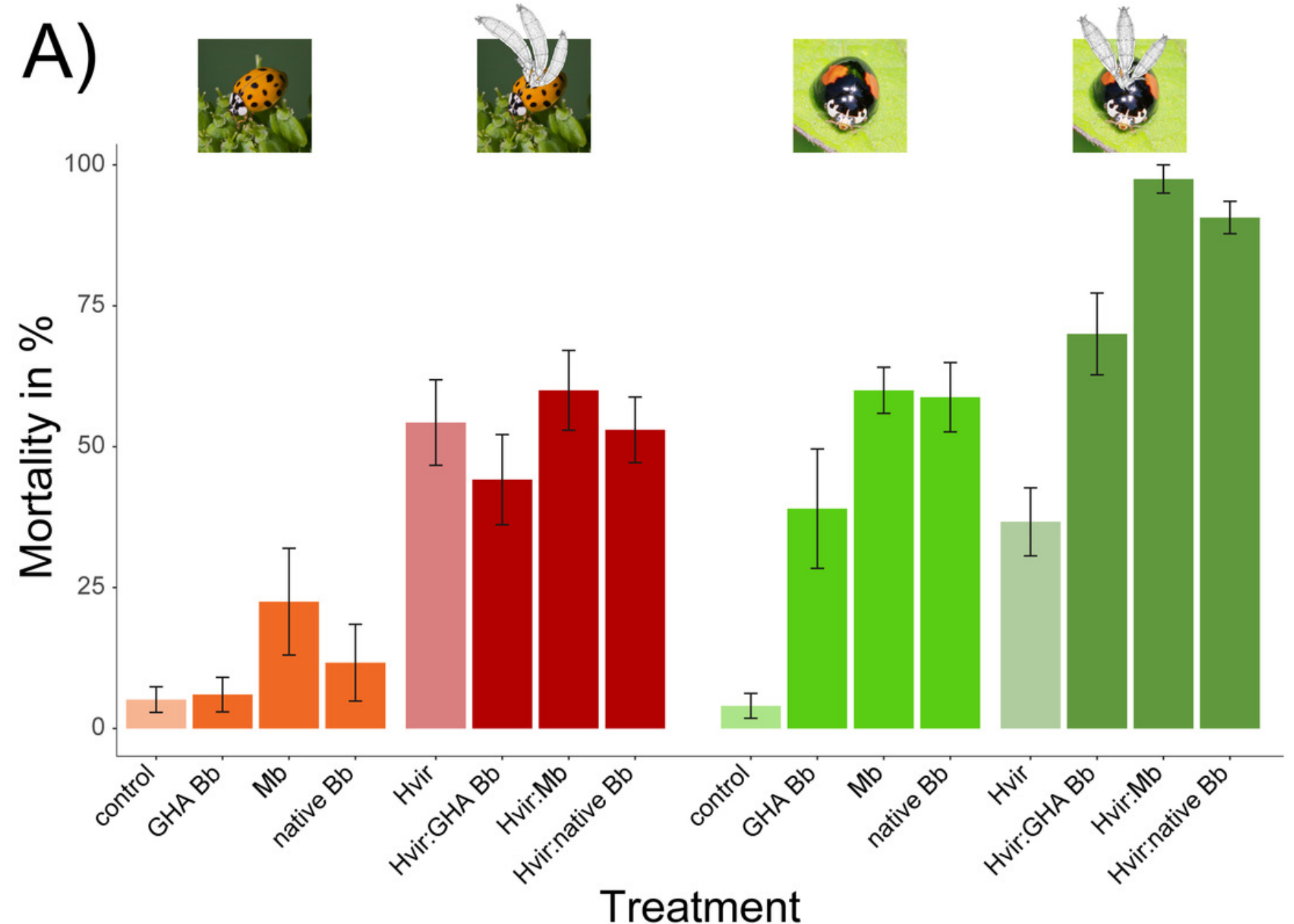

B)

Hvir:native $\mathrm{Bb}$ Hvir:GHA Bb

Hvir:Mb

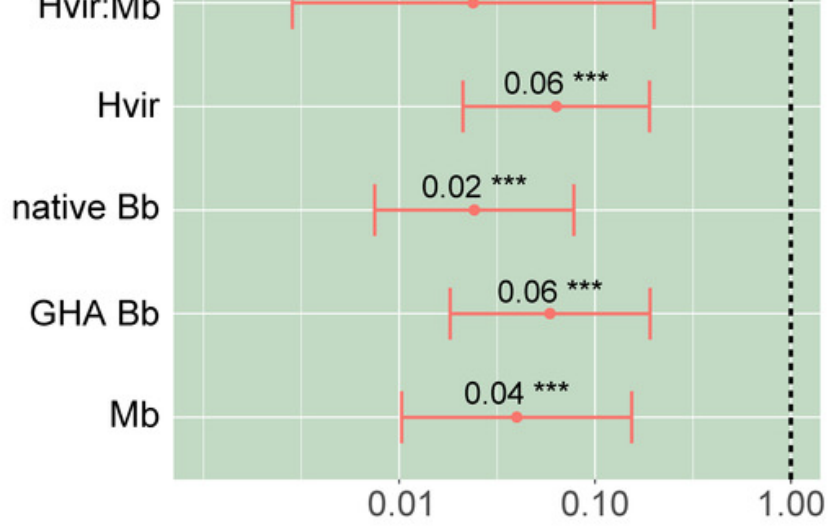

Odds Ratios

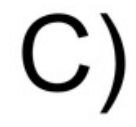

Hvir:native $\mathrm{Bb}$

Hvir:GHA Bb

Hvir:Mb

Hvir

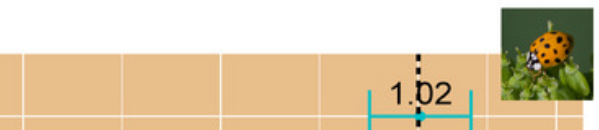

native $\mathrm{Bb}$

$\mathrm{GHA} \mathrm{Bb}$

$\mathrm{Mb}$

0.16 **

0.53

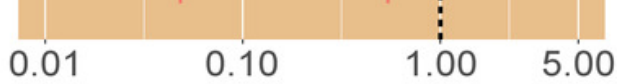

Odds Ratios 


\section{Figure 2}

Effect of Hesperomyces-infection and co-infection with Hesperomyces and Beauveria bassiana on the survival of ladybirds.

A) Survival of Hesperomyces-positive ladybirds without dual infection. B) Hesperomycespositive ladybirds co-infected with GHA strain of Beauveria bassiana. C) Hesperomycespositive ladybirds infected with native Beauveria bassiana. The survival of ladybirds is significantly different between Harmonia axyridis and Olla v-nigrum with the dual infection treatments.

A) Hvir

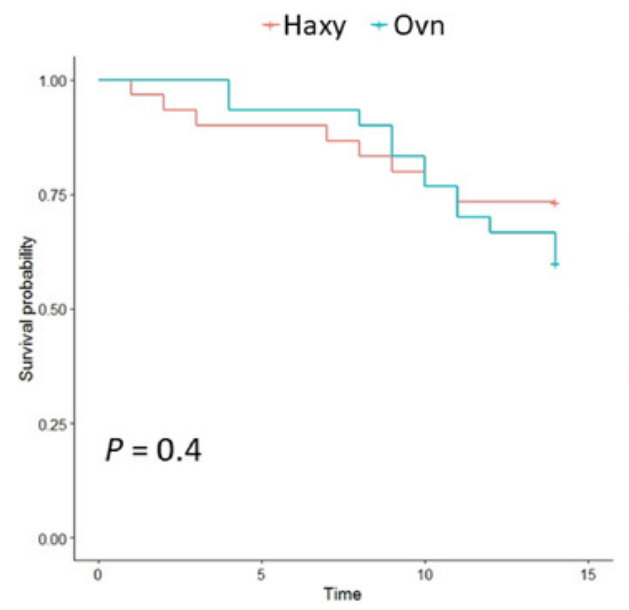

B) Hvir:GHA Bb

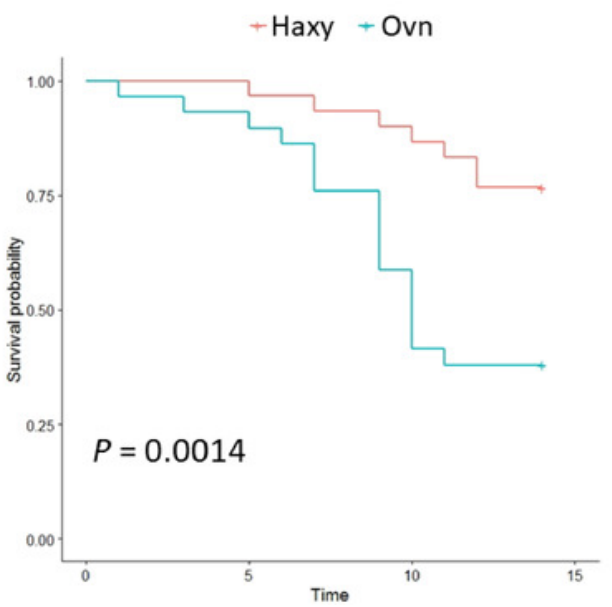

C) Hvir:native $\mathrm{Bb}$

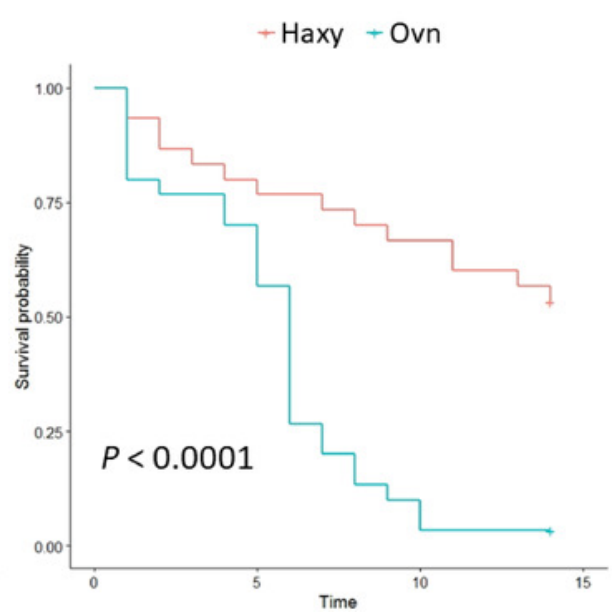




\section{Table $\mathbf{1}$ (on next page)}

Results of our modeling approach for Harmonia axyridis and Olla v-nigrum.

Summary of parameters corresponding to Hesperomyces virescens infection and respective interactions with GHA Beauveria bassiana, native B. bassiana, and Metarhizium brunneum. 
1 Table 1. Results of our modeling approach for Harmonia axyridis and Olla v-nigrum.

2 Summary of parameters corresponding to Hesperomyces virescens infection and respective

3 interactions with GHA Beauveria bassiana, native B. bassiana, and Metarhizium brunneum.

4

\begin{tabular}{llrrrrr}
\hline Harmonia axyridis & & Estimate & Std. Error & $\mathrm{z}$ value & $P$-value & \\
\hline (Intercept) & & 2.977 & 0.563 & 5.284 & $<\mathbf{0 . 0 0 1}$ & $* * *$ \\
\hline He. virescens infection & & -3.147 & 0.518 & -6.071 & $<\mathbf{0 . 0 0 1}$ & $* * *$ \\
\hline He. virescens-negative & GHA Bb & -0.179 & 0.630 & -0.284 & 0.777 & \\
& Native Bb & -0.642 & 0.585 & -1.098 & 0.272 & \\
& $\mathrm{Mb}$ & -1.820 & 0.618 & -2.946 & $\mathbf{0 . 0 0 3}$ & $* *$ \\
\hline He. virescens-positive & GHA Bb & 0.454 & 0.305 & 1.487 & 0.137 & \\
& Native Bb & 0.024 & 0.302 & 0.080 & 0.936 & \\
& $\mathrm{Mb}$ & -0.330 & 0.406 & -0.811 & 0.417 & \\
\hline Olla v-nigrum & & Estimate & Std. Error & $\mathrm{z}$ value & $P$-value & \\
\hline (Intercept) & & 3.407 & 0.609 & 5.598 & $<\mathbf{0 . 0 0 1}$ & $* * *$ \\
\hline He. virescens infection & & -2.757 & 0.560 & -4.925 & $<\mathbf{0 . 0 0 1}$ & $* * *$ \\
\hline He. virescens-negative & $\mathrm{GHA} \mathrm{Bb}$ & -2.831 & 0.599 & -4.728 & $<\mathbf{0 . 0 0 1}$ & $* * *$ \\
& $\mathrm{Native} \mathrm{Bb}$ & -3.723 & 0.597 & -6.234 & $<\mathbf{0 . 0 0 1}$ & $* * *$ \\
& $\mathrm{Mb}$ & -3.222 & 0.689 & -4.676 & $<\mathbf{0 . 0 0 1}$ & $* * *$ \\
\hline He. virescens-positive & $\mathrm{GHA} \mathrm{Bb}$ & -1.591 & 0.390 & -4.084 & $<\mathbf{0 . 0 0 1}$ & $* * *$ \\
& $\mathrm{Native} \mathrm{Bb}$ & -2.988 & 0.470 & -6.364 & $<\mathbf{0 . 0 0 1}$ & $* * *$ \\
& $\mathrm{Mb}$ & -3.734 & 1.084 & -3.444 & $<\mathbf{0 . 0 0 1}$ & $* * *$ \\
\hline
\end{tabular}

5 